\title{
BMJ Open Physical activity and health promotion for nursing staff in elderly care: a study protocol for a randomised controlled trial
}

\begin{abstract}
Ann-Kathrin Otto (D) , ${ }^{1}$ Juliane Pietschmann, ${ }^{2}$ Luisa-Marie Appelles, ${ }^{3}$ Michael Bebenek, ${ }^{4}$ Laura L Bischoff, ${ }^{1}$ Claudia Hildebrand, ${ }^{3}$ Bettina Johnen, ${ }^{5}$ Thomas Jöllenbeck, ${ }^{2}$ Wolfgang Kemmler, ${ }^{4}$ Thomas Klotzbier, ${ }^{5}$ Heide Korbus, ${ }^{5}$ Julian Rudisch, ${ }^{6}$ Nadja Schott, ${ }^{5}$ Daniel Schoene, ${ }^{4}$ Claudia Voelcker-Rehage, ${ }^{6}$ Oliver Vogel, ${ }^{7}$ Lutz Vogt, ${ }^{7}$ Matthias Weigelt, ${ }^{2}$ Jan Wilke, ${ }^{7}$ Katharina Zwingmann, ${ }^{8}$ Bettina Wollesen ${ }^{9}$
\end{abstract}

To cite: 0 tto A-K,

Pietschmann J, Appelles L-M, et al. Physical activity and health promotion for nursing staff in elderly care: a study protocol for a randomised controlled trial. BMJ Open 2020;10:e038202. doi:10.1136/ bmjopen-2020-038202

- Prepublication history for this paper is available online. To view these files, please visit the journal online (http://dx.doi. org/10.1136/bmjopen-2020038202).

Received 10 March 2020 Revised 22 July 2020 Accepted 21 August 2020

\section{Check for updates}

(C) Author(s) (or their employer(s)) 2020. Re-use permitted under CC BY-NC. No commercial re-use. See rights and permissions. Published by BMJ.

For numbered affiliations see end of article.

\section{Correspondence to} Ann-Kathrin 0tto; ann-kathrin.otto@uni-hamburg. de

\section{ABSTRACT}

Introduction Nursing staff is burdened by high workload and stress. Furthermore, heavy lifting, as well as transferring nursing home residents, cause lumbar tissue damage and back pain. Exercise intervention studies to reduce work-related problems are rare and the evidence for efficacy of studies among nurses is limited. Studies including targeted analysis of requirements are necessary to generate effective recommendations and tailored interventions for health promotion programmes. The purpose of this multicentred intervention study is to identify work-related problems, to implement health promotion programmes and to evaluate their effectiveness. Methods and analysis A randomised controlled trial will be conducted, including a total of 48 nursing home facilities in eight regions of Germany with an estimated sample size of 700 nurses. Standardised ergonomics and posture training (10 weeks, once a week for 20-30 min) and subsequently, back-fitness training (12 weeks, once a week for $45-60 \mathrm{~min}$ ) will be administered. Following the implementation of standardised health promotion programmes, further demand-oriented interventions can be implemented. The perceived exposure to work-related demands, work-related pain in different parts of the body, health-related quality of life, perceived stress, workrelated patterns of behaviour and experience, presentism behaviour, work environment as well as general needs and barriers to health promotion, will be assessed at baseline (pre-test), at 10 weeks (post-test, after ergonomics training), at 22 weeks (post-test, after back-fitness training) and at 34 weeks of the programme (follow-up). Ethics and dissemination The study was reviewed and approved by the local ethics committee of the University of Hamburg (AZ: 2018_168). The results of the study will be published in open-access and international journals. Furthermore, the results will be presented in the participating nursing homes and at national and international conferences.

Trial registration number DRKS.de (DRKS00015241).

\section{INTRODUCTION}

The demographic change causes an increasing number of elderly people to reside

\section{Strengths and limitations of this study}

- This trial will provide novel information about the efficacy of a multifactorial intervention programme for nurses in elderly care.

- Recommendations for effective health promotion programmes in nursing home facilities can be derived from the results of the current study and made available for practical use.

- Participants were asked to complete questionnaires during working hours, although the time pressure can influence the quality of the answers.

in a nursing home facility, characterized by multimorbidity at high risk of disability. ${ }^{1}$ In 2018, only 421287 full-time nurses were employed in Germany, ${ }^{2}$ although the demand is higher. Nonetheless, nursing home facilities face difficulties when hiring and integrating new staff. ${ }^{34}$ Subsequently, this leads to high levels of work-related strain and stress, ${ }^{5-7}$ resulting in 25.5 days of inability to work per employee, exceeding the average population by 10 days. ${ }^{8}$

Compared with other professions, nursing staff have a six times higher prevalence of back pain. ${ }^{9}$ The basic assumption is that there are multiple causes for back pain. The interaction of biological, psychological and social factors leads to long-term maintenance of back pain. ${ }^{10}$ Research has shown that frequently performed transferring tasks can cause lumbar tissue damage and back pain when nursing staff are under time pressure and operate in awkward body postures (eg, turning and holding nursing home residents). ${ }^{5} 1213$ Unsurprisingly, lumbar dysfunctions and the increased risk of back pain have 
also been associated with high demands, burnout, low control and a lack of social prestige. ${ }^{14}$

Several studies have investigated the effect of exercise interventions on back pain in nursing staff in all settings. ${ }^{13}{ }^{15-20}$ However, effective exercise intervention studies to reduce work-related problems in nursing home staff are rare and therefore, today, little is known about the efficacy of interventions to reduce back pain in nursing staff in elderly care. In addition, studies have shown that successful interventions employed a bottom-up approach and took setting specific factors, as well as work-related burdens, wishes, needs and barriers of the target group into account. ${ }^{1721} 22$ For other professions, it has already been proven that programmes combining ergonomics training and the promotion of physical resources can effectively reduce back pain. ${ }^{23}$ First, studies could also show effects in the area of nursing settings, however, according to a systematic review, there is a lack of highquality randomised controlled trials. ${ }^{21}$ Skargren and Oberg ${ }^{15}$ demonstrated in a prospective cross-over study that moderate exercises, including stretching exercises and strength exercises with intervals of cardiovascular capacity exercises, reduce musculoskeletal symptoms and increase the cardiovascular capacity and muscular strength among nursing staff. On the other hand, Horneij et $a l^{16}$ have established in a prospective randomised study that an individually designed physical training programme, combined with a workplace stress management training among nursing staff in home care had no effect on neck, shoulder and low back pain. In line with this study, Ewert et $a l^{17}$ observed in a randomised controlled parallelgroup trial small to moderate effects of a multimodal programme including psychological, segmental exercises and ergonomic training sessions, compared with a general physical exercise programme, designed to reduce chronic low back pain. Based on the current evidence, it is difficult to draw firm conclusions about the efficacy of interventions for low back pain, also due to a lack of high quality randomised controlled trials. ${ }^{21}$

In order to design and implement effective interventions, it is necessary to engage employees in the planning and implementation of health promotion programmes. Thereby, the differentiated documentation of workrelated risks, needs, wishes and barriers is relevant, as this is considered as a key factor for the successful implementation of health promotion programmes. ${ }^{22}{ }^{24}$ This ensures that sustainable and long-term behavioural modifications can be initialised and the motivation of the employees can be maintained. ${ }^{25}$ Additionally, the quality of implementation and theory-based programmes are responsible for the success. ${ }^{26}{ }^{27}$ Besides, Rongen et al showed that workplace interventions in small groups are more effective than those in large groups. ${ }^{28}$

To use the suggestions of these previous studies, the BASE concept-a prevention programme to avoid and reduce mental stress and musculoskeletal diseasescan be applied. ${ }^{24}$ BASE stands for B, 'Bedarfsbestimmung' (requirements); A, 'Arbeitsplatzorganisation' (organisation of work); S, 'Schulung des arbeitsbelastungsverträglichen Alltagshandeln' (coaching preventive behaviour at work) and E, 'Eigenverantwortung und Selbstwirksamkeit' (self-responsibility and self-efficacy). The programme considers the working conditions, the organisational and social environment of the nursing home facilities and involves employees in the assessment of working health risks. Within this framework, wishes and barriers for health promotion programmes are recorded to achieve high participation rates. ${ }^{25}{ }^{26}$ This approach of multidimensional assessment has proven to be successful to achieve positive health effects. ${ }^{29}$ For instance, implementing BASE, including ergonomics and posture training in a logistics department resulted in reduced low back pain and decreased dysfunctional lifting behaviour. ${ }^{22}$

The overall objectives of this randomised controlled trial are (1) the evaluation of setting specific, individual and organisational context variables (subjectively perceived exposure to work-related demands, work-related pain in different parts of the body, health-related quality of life, perceived stress, work-related patterns of behaviour and experience, presentation behaviour, work environment, general needs and barriers to health promotion; infrastructure of the facility, social environment) and, (2) the review of the BASE concept in the nursing home setting. Following the implementation of standardised health promotion programmes further demand-oriented interventions can be implemented, considering the general needs and participants feedback after the standardised interventions. We hypothesise that the BASE programme, here consisting of a 10-week ergonomics and posture training and a 12-week back-fitness programme, including the setting specific requirements, will lead to improvements, especially in terms of perceived exposure to work-related demands (modified Slesina Questionnaire), work-related pain in different parts of the body during the last 7 days (Nordic Questionnaire) and healthrelated quality of life (Health Survey (SF-12)).

\section{METHODS AND ANALYSIS}

The SPIRIT (Standard Protocol Items: Recommendations for Interventional Trials) statement ${ }^{30}$ was used as a guideline for this protocol paper.

\section{Study design}

This is a multicentred randomised controlled trial of an individually tailored multifactorial intervention for nursing staff. The study is part of the project 'Prevention and occupational health in long-term care (PROCARE)', which addresses the living and working situations of staff and residents in the nursing home setting. ${ }^{31}$ The nurses will be randomised to an intervention group and waitinglist control group. Both groups will receive the intervention. The waiting-list control group will start with the intervention after 10 weeks. The questionnaire data will be assessed at baseline (pre-test), at 10 weeks (post-test, 


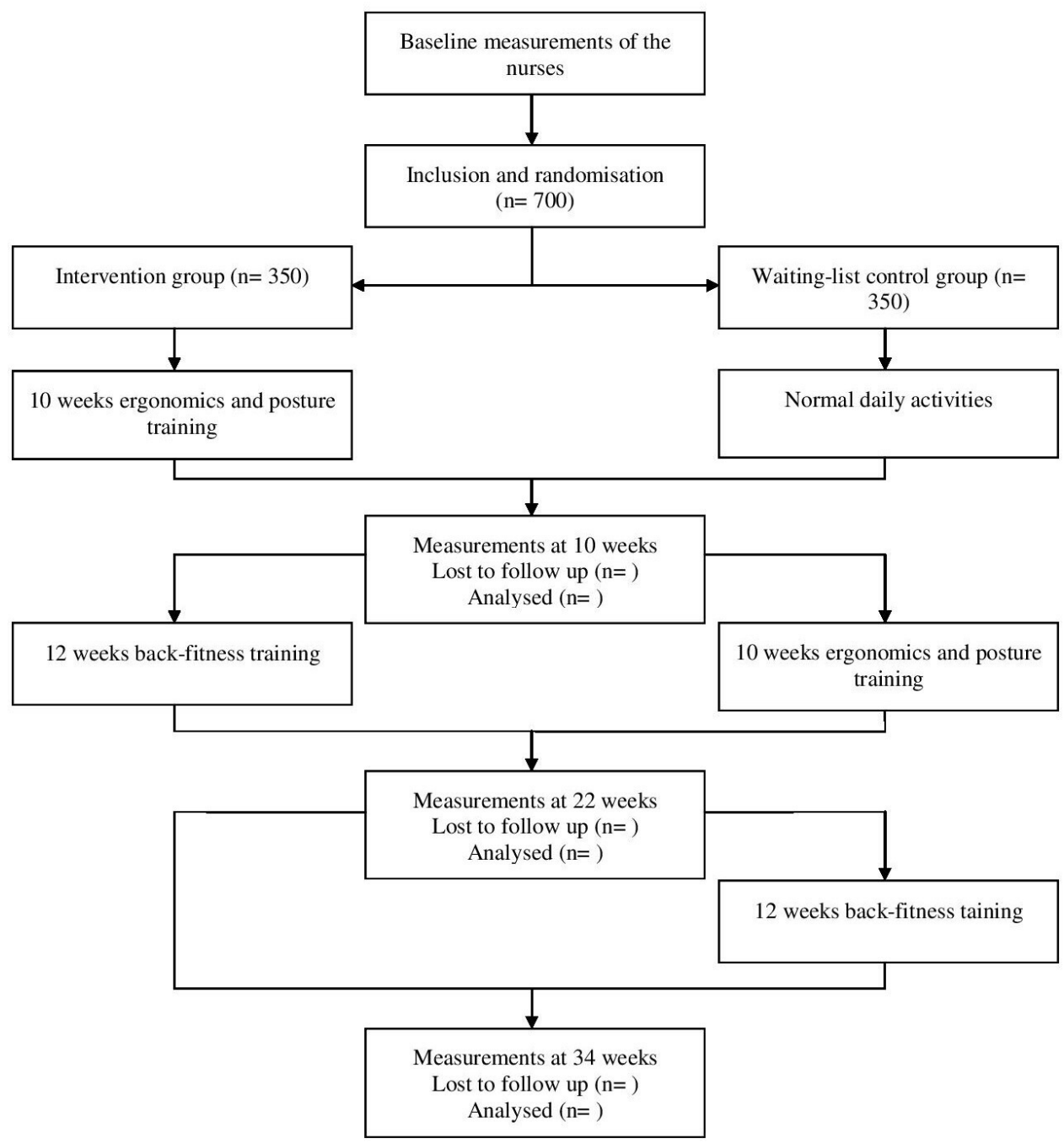

Figure 1 Flow diagram.

after ergonomics training and posture training), at 22 weeks (post-test, after back-fitness training) and at 34 weeks of the programme (follow-up) (see figure 1).

The trial is conducted in agreement with the principles of the Declaration of Helsinki and the guidelines of good clinical practice. The study was approved by the local ethics committee of the University of Hamburg (AZ: 2018_168) and is registered at DRKS.de.

\section{Setting}

This study will include 48 nursing home facilities located in one of eight cities and their surroundings throughout Germany (Bremen, Chemnitz, Frankfurt, Hamburg, Karlsruhe, Nuremberg, Paderborn and Stuttgart). In all eight regions, six facilities were chosen. The infrastructure forms the basis for the selection of the nursing home facilities at each intervention side. This includes the number of nursing places, the urban or rural district, the number of employees and the socioeconomic status. The intervention sides participating in this study have been selected in order to analyse the programmes' applicability under a wide range of conditions.

\section{Patient and public involvement}

No patients will be involved in this study. We will integrate the nurses into the programme development by recording their working health risks, wishes, needs and barriers with a questionnaire. The results will be taken into account to plan and implement health promotion interventions. In addition, the results will be disseminated in the participating nursing home facilities during information events and/or in the form of written mail drops.

\section{Participants}

All nursing staff of the recruited nursing home facilities will be asked to participate in the context of comprehensive information events.

\section{Inclusion criteria}

The randomised controlled trial will include nursing staff of nursing home facilities, which provide written informed consent, whereas other professionals of nursing home facilities will be excluded. The estimated participation rate is $30 \%$. 


\section{Assignment of intervention}

To avoid selection bias, the allocation of the nurses in intervention group and waiting-list control group will be assigned randomly by lot by the director of the study. Data collection will be done by blinded assessors in a strictly pseudonymised form to guarantee a blinded data analysis. The multifactorial intervention programme follows a standardised protocol to avoid performance bias. All participant information and data will be stored securely and pseudonymised with the aid of a code and after completion of the data collection is replaced by a pure combination of numbers (eg, PA1). Personalised data are only available for the director of the study.

\section{Outcome measures}

The assessment will focus on a battery of five standardised instruments. Besides collecting questionnaire data, demographic characteristics, such as age, gender and job title, will be assessed.

\section{Primary outcomes}

To evaluate the effectiveness of the intervention programme, self-reported perceived exposure to workrelated demands (modified Slesina Questionnaire), workrelated pain in different parts of the body during the last 7 days (Nordic Questionnaire), health-related quality of life (SF-12), perceived stress and work-related patterns of behaviour and experience will be assessed as primary outcomes.

The Questionnaire for Subjective Assessment of Workplace Exposure (modified Slesina Questionnaire) comprises 20 items about work-related demands (physical, psychological and environmental factors), the intensity of daily work, as well as corresponding perceived exposure. ${ }^{32}$ The questionnaire assesses the frequency of demand factors (frequently, seldom/never) and the subjectively perceived amount of physical or mental perceived exposure (yes, no). These 20 items included heavy physical tasks, awkward postures, standing, overhead working, holding heavy loads, carrying heavy loads, lifting heavy loads, pulling/pushing heavy loads, awkward static postures, the lack of physical activity, sitting, walking/ running, dexterity, visual detail distinction, poor illumination, IT problems, pressure to perform, deadline pressure, time pressure and shift work. ${ }^{33}$ The questionnaire is used for analyses by the Federal Institute for Occupational Safety and Health (BAuA). The use of this questionnaire will allow us to set the data of the project in relation to nationwide norm data.

The Questionnaire on Musculoskeletal Complaints (Nordic Questionnaire) is an evaluation tool for work-related pain in different body parts (neck, shoulder, elbows, hands, lumbar spine, spine, hips, knees and feet) during the last 12 months and the last 7 days. ${ }^{34}$ Participants rate their impairments on a two-point scale (yes, no), by responding to nine items regarding the relevant body parts. They were asked the following: 'Have you had any complaints or pain in the following body parts during the last 12 months?', 'Were you unable to do your normal work at any time in the last 12 months because of the complaints?' and 'Have you had any complaints during the last 7 days?'. The questionnaire is considered valid and reliable in German version. ${ }^{34}$

The SF-12 is an economic short form of the SF-36 and a disease-independent questionnaire to measure the health-related quality of life, consisting of 12 items. ${ }^{35}$ The summary scales were each derived from four subscales: Physical Health consists of physical functioning, role limitations due to physical health problems, bodily pain and general health, whereas Mental Health is summarising the subscales vitality (energy/fatigue), social functioning, role limitations due to emotional problems and mental health (psychological distress and psychological wellbeing). They were asked for example, the following: 'How would you describe your state of health in general?', or 'To what extent have you been hampered in the past 4 weeks in performing your daily activities at home and at work?'. It is considered valid and reliable. In terms of reliability, the internal consistency (Cronbach's $\alpha$ ) for the scale of physical function in both healthy and sick persons is between 0.77 and 0.93 , the physical role function scale between 0.74 and 0.89 , the pain scale between 0.73 and 0.85 , the general health perception scale between 0.57 and 0.75 , the scale vitality between 0.78 and 0.84 , the social function of the scale between 0.64 and 0.88 , the scale emotional role function between 0.77 and 0.94 and that of the scale mental well-being between 0.78 and $0.88 .^{36}$ The German version of the SF-12 was translated and validated in accordance with the standards of the International Quality of Life Assessment Group. ${ }^{37}$

The 12-item screening subscale (SSCS) of the Trier Inventory for Chronic Stress (TICS) provides information about perceived stress within the last 3 months, covering five aspects of stress, including chronic worrying, workrelated and social overload, excessive demands and lack of social recognition. Participants can rate for example, the following statements: 'Fear of something unpleasant happening', or 'I try in vain to get recognition for good performance'. Stress frequency are rated on a four-point scale $(0=$ never, $1=$ rarely, $2=$ sometimes, $3=$ often, $4=$ very often). The scale is considered a reliable measuring instrument; the internal consistency (Cronbach's $\alpha$ ) is between 0.84 and $0.91 .^{38}{ }^{39}$ The Trier Inventory for Chronic Stress was validated in the German version. ${ }^{39}$

The Questionnaire on Work-Related Behavior and Experience Patterns (AVEM) contains a total of 44 items. As a multidimensional personality diagnostic procedure, the AVEM-44 collects self-assessments within eleven dimensions (scales), which can be assigned to three content areas: professional commitment, resistance towards stress and emotional well-being. The participants can for example, make a statement about the following items: 'For me, work is the most important part of my life', 'After work I can switch off without any problems', or 'Whatever I do, it has to be perfect'. The response options are presented as a five-point scale ranging from 1 ('I strongly 
disagree') to 5 ('I strongly agree). The AVEM subscales can be used to classify four types of behavioural and experiential patterns: Pattern G 'Health', Pattern S 'Unambitious', Risk pattern A 'Overexertion' and Risk pattern B 'Burnout'. ${ }^{40}$ It is considered valid and reliable in German version. The internal consistency (Cronbachs $\alpha$ ) is between 0.75 and $0.84 .^{41}$

\section{Secondary outcomes}

The state of health, sports and eating habits, presentism behaviour, general needs and barriers to health promotion, as well as work environment will be assessed as secondary outcomes.

The Questionnaire on Resources contains 12 self-developed questions regarding the state of health, the kind and duration of weekly and daily activity, the eating habits and the stress level, for example, 'How satisfied are you with the state of health?', 'How many hours per week do you spend exercising/playing sports?', 'How would you rate your dietary behaviour?', or 'How many times have you been calm und serene in the last 4 weeks?'. The response options are presented as a five-point scale. The questionnaire is based on the validated SF- $12,{ }^{35}$ the Bone-Specific Physical Activity Assessment Instrument ${ }^{42}$ and the WHO guidelines 'Sugars intake for adults and children'. ${ }^{43}$

The Questionnaire on Presentism provides information on the issue of presentism and emphasises its reasons and consequences. They were asked the following selfdeveloped questions, for example, 'How often have you been working in the last 12 months despite deteriorating health, although you felt you had to stay at home?', 'With what kind of symptoms did you appear at work?', or 'Why did you come to work sick?'. These items are based on the Health and Work Performance Questionnaire. ${ }^{44}$

The Questionnaire on general Needs and Barriers to Participate in Health Promotion Programmes covers the wishes regarding health promotion programmes and barriers preventing from participating. Examples of self-develop items are: 'What kind of exercise promotion programme would you be interested in?' (response options included strength training, endurance training, relaxation training, others), or 'If you currently are not attending any health promotion programmes, please mark with a cross the barriers that are possibly keeping you from doing so'. (answer options were eg, 'I feel to strained to attend any physical activity or other additional programme', 'Additional activities are too time consuming' or 'I don't know of any suitable offers'). The questionnaire was developed as part of a project in which 48 companies took part. ${ }^{45}$

The self-developed Questionnaire on Work Environment offers the opportunity to specify the work environment, regarding colleagues (items: eg, 'The working atmosphere is impersonal', or 'If someone has difficulties at work, they will certainly be helped by colleagues'), supervisors (items: eg, 'Good work is recognised by our supervisors accordingly', or 'The supervisors respond to concerns and complaints') and the areas of information- and communication exchange (items: eg, 'We are sufficiently informed about important things and processes in our company', or 'The information about the planned implementation of novelties is so sufficient that we know exactly what to expect'). Participants can indicate their answer on a sixpoint-scale. The questionnaire was developed in cooperation with nursing home facilities.

\section{Intervention}

The multifactorial intervention programme consists of a standardised ergonomics and posture training and a standardised back fitness training. ${ }^{25}$ The programme was developed on the basis of earlier study results, the validated BASE concept, ${ }^{22} 24$ as well as workplace observations. Interventions in each participating facility will start with a workplace observation of specific basic ergonomic condition, in order to take these conditions, such as existing lifting, aids into account during specific units of the training programme. Both trainings will be supervised and guided by certified exercise scientists or physiotherapists, who are trained to work according to a standardised manual.

The ergonomics-training and posture-training programme takes place over a period of 10 weeks, once a week for a duration of 20-30 min. The sessions include six to eight participants per group. It includes the learning of different techniques, in order to deal with physical stress at the workplace and to compensate in a stresscompatible manner. The training programme is divided into the following sections:

- Exercises for movement and body perception in the work process.

- Reflection of one's own movement and work behaviour.

- Instructions for motion optimisation.

- Instructions for independent compensation exercises.

- Personal health promotion measures. ${ }^{26} 27$

Each session sets priorities for different work-related tasks. In this way, relevant topics are discussed and practiced in detail. The movement experience is comprised of three components: body awareness, recognition of dysfunctional movements and understanding positive and negative work behaviour. The reflection of the movement experience, the adaption to the working conditions, the repeated implementation and testing in the work situation should positively influence attitudes, intentions and behaviours. The intention of the course is that afterwards, employees are able to independently recognise and influence possible health-related resources, but also potential dangers. The exercises for the individual areas are repeated for in-depth study and in part supplemented by new exercises. This course is not about being introduced to many different exercises and methods, but to learn and vary simple and effective exercises, which can be easily integrated into everyday life. Positive and joyful movement experiences should be collected, in order to help to remove barriers and deepen and internalise knowledge and different ways of execution, as well as develop selfhelp strategies for back problems. 
The back-fitness programme is carried out over a period of 12 weeks, once a week for $45-60 \mathrm{~min}$. It includes the components mobility, coordination, strength and relaxation. Strength training is divided into three phases, each lasting 4 weeks. In each phase, a progression is planned. This will be ensured by adjusting the frequency, difficulty and range of motion/intensity of the exercises.

In order to obtain a verifiable structure, the back-fitness programme is divided as follows:

1. Mobility training (5-10 $\mathrm{min})$.

2. Coordination training (10-15 min).

3. Strength training $(30-40 \mathrm{~min})$.

4. Relaxation (5-10 min).

In the back-fitness programme, the joints that tend to stiffen are mobilised and theoretical knowledge about the execution of conscious movements and the targeted control of the musculature is imparted. Strength training focuses on the back, shoulder and neck muscles. The abdominal arm and leg muscles are also strengthened.
In the relaxation part, different techniques will be used, such as yoga, progressive muscle relaxation and breathing exercises. Exercises will be performed at a moderate intensity (5-6 on Borg CR10 Scale ${ }^{46}$ ). To improve adherence and to promote retention, the therapists will give explanations about the purpose of the intervention and the possible benefits of the exercises. Attendance of each participant will be recorded by attendance lists and reasons for drop out will be documented. In addition, compliance of participants will be collected by a short questionnaire after both trainings.

The interventions will take place at the facilities during working hours to reach as many employees as possible. Concerning this, early organisation and consultation with the supervisors is essential, in order to adjust the working hours of the employees accordingly, and at the same time, to ensure residents care.

For the schedule of enrolment, interventions and assessments, see table 1 .

Table 1 Schedule of enrolment, interventions and assessments

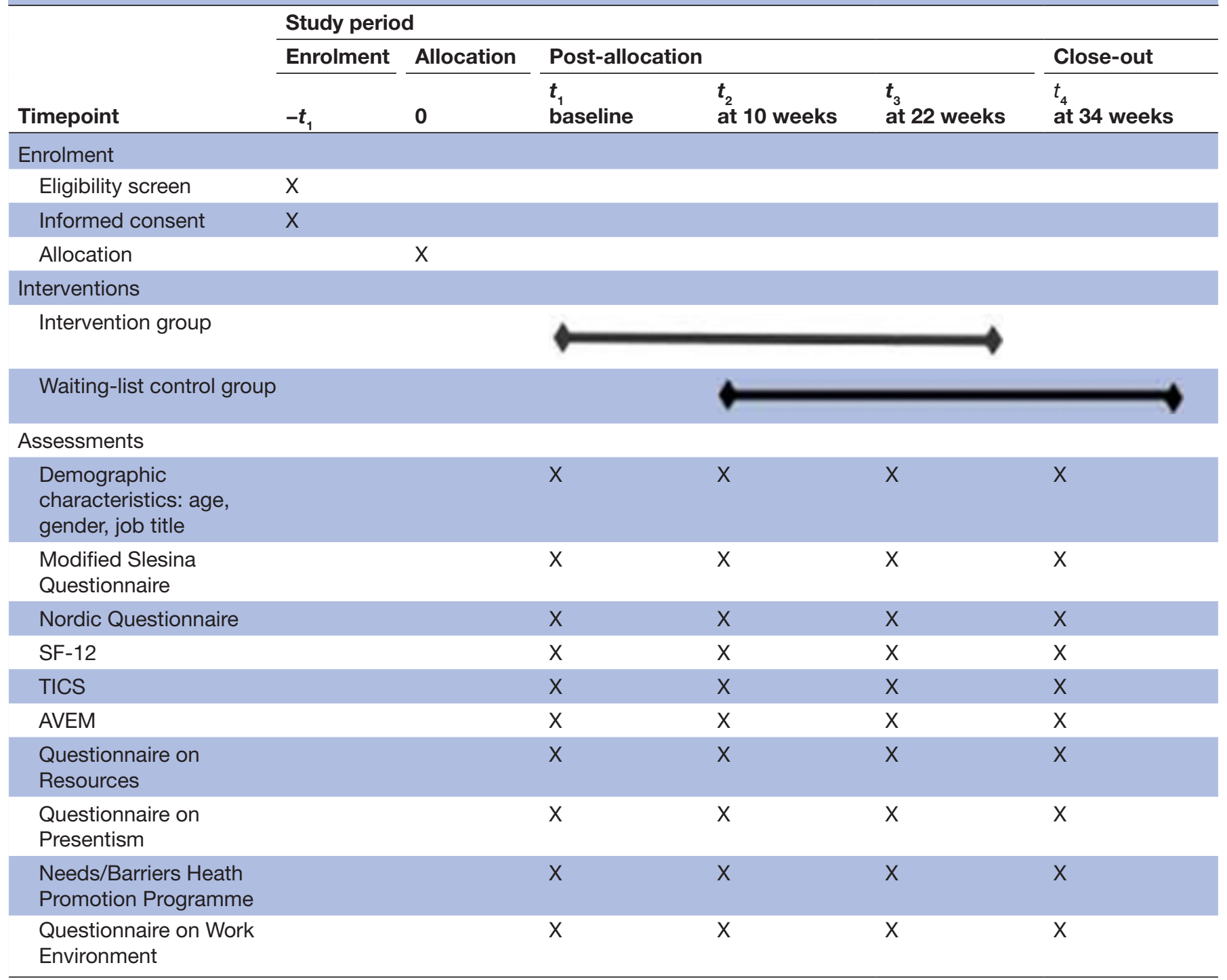

AVEM, Questionnaire on Work-Related Behavior and Experience Patterns; SF-12, Health Survey; TICS, Trier Inventory for Chronic Stress. 


\section{Statistical analysis}

The trial will adhere to the 'Consolidated Standards of Reporting Trials statement: extension to cluster randomised trials' ${ }^{47}$ Data will be analysed on an intentionto-treat basis, based on staff group.

The study will integrate a comparison between the intervention and the waiting-list control group, as well as a comparison between the training programmes. In addition, participation rate will be integrated into the analysis.

Sample characteristics will be explored using descriptive statistics, and standard analyses adjustments will be made to adjust for baseline differences between groups, should such exist. In cases of missing data, sensitivity analyses will be performed to compare results with complete case analyses, and different options for imputation will be considered. Differences between intervention and controls will be tested with $\chi^{2}$ tests for categorical variables and independent sample t-test for continuous variables.

Because of the cluster randomisation multilevel models with the nursing home as random effect, general linear mixed-model regressions will be applied for the statistical analyses of primary and secondary endpoints. These models will be adjusted by baseline value and potential confounding variables, for example, location of the nursing home (urban vs country), and staff field of working. $p$ values of $<0.05$ will be regarded as statistically significant and effect sizes of $>0.3$ will be regarded as clinically significant. Where appropriate, $95 \%$ CI will also be reported along with the $\mathrm{p}$ values.

Data from the trial will be analysed using SPSS Statistics for Windows V.25.0 (IBM, Armonk, New York, USA).

\section{Power calculation}

The required sample size was calculated with $G^{*}$ Power (V.3.1.9.2, Heinrich Heine University of Duesseldorf). ${ }^{48}$ The mean overall SF-12 score in the population is 50 with an SD of $10 .{ }^{49}$ Studies estimated a minimal clinically important difference (MCID) of $\geq 3$ points for the SF-12.

In total, 700 participants will need to be recruited in expectation that 560 will provide data throughout the 34 weeks. This sample size will ensure $90 \%$ power to detect MCID of 3 at the primary time point of 34 weeks follow-up, given a 5\% two-tailed significance level. Randomisation is by practice, so this sample size calculation was inflated to correct for an intracluster correlation coefficient (adjusted intracluster correlation coefficient of 0.005), varying practice size recruitment was taken into account (including coefficient of variation of 0.5 ) and inclusive of 1.25 adjustments for repeated-measures design and $20 \%$ dropout allowance. A medium effect size based on the literature will be expected. ${ }^{50}$

\section{Monitoring}

There will be no data monitoring committee responsible for data monitoring, interim analyses and auditing, as no adverse events are expected. If a negative reaction is observed during the measurements and training interventions, the trained project staff will intervene.
Furthermore, grant holders are responsible for data audits every 5 months as members of PROCARE advisory board.

\section{Ethics and dissemination}

The study was reviewed and approved by the local ethics committee of the University of Hamburg (AZ: 2018_168). The results of the study will be published in open-access and international journals. Furthermore, the results will be presented in the participating nursing homes and at national and international conferences.

\section{DISCUSSION}

So far, a health management is not regularly available in nursing homes. Often there is a lack of financial and time resources and a lack of consideration of setting specific needs. Target groups, who are physically strained, often have to be motivated to participate in health promotion activities. In particular, the challenges in developing effective interventions in nursing home facilities are the structural framework conditions, shift work and the heavily burdened nursing staff. Exercise intervention studies to reduce work-related problems in nursing staff are rare and the evidence for efficacy of intervention studies among nursing staff is limited. ${ }^{15} 161851$ High quality studies are required to provide recommendations for health promotion programmes for nursing staff in elderly care, in order to address the increasing demands and the lack of skilled personnel. The BASE concept, which is central to the method of this study, includes all necessary factors to implement a successful health promotion programme in this context. ${ }^{31}$ However, it remains unclear whether this participatory approach is as successful as in other BASE interventions. Wollesen $e t a t^{22}$ could demonstrate sustainable changes in work-related problems, when implementing the BASE concept in the logistic, industrial and office sector.

Therefore, the current study offers the opportunity to implement effective interventions using the multidimensional approach of the BASE concept, which engages employees in the planning and implementation of health promotion programmes. Moreover, optionally further demand-oriented interventions can be implemented afterwards to motivate participants to engage in additional physical activities and thus, promote a sustainable healthy and active lifestyle.

The standardised multifactorial intervention programme, which is implemented based on the nursing home facilities' infrastructure, as well as organisational and social environment, will help to increase the compliance to the intervention and to reduce work-related problems like back pain in nursing staff.

As a major benefit of the study, more specific recommendations for effective prevention measures in nursing home facilities can be derived from the results of the study and made available for wider implementation. 
Nevertheless, there are some limitations in the study: The participants will be asked to fill in the questionnaires in the context of information events during working time. It will take approximately $30 \mathrm{~min}$ to complete the questionnaire. Lack of time and time pressure can influence the quality of the answers. In order to avoid this, several information events will take place if necessary. This ensures that the nurses do not fill out the questionnaires at the same time, thus guaranteeing the care of the residents and to avoid time pressure. Moreover, another confounding factor could be the interchange about the health promotion programmes between the nurses in different groups, leading to changes in the waiting-list control group. This is a fundamental problem in the implementation of health promotion programmes, but the design has already been used in other BASE studies, reporting greater effects in the intervention group than in control group. ${ }^{22}$ In addition, work culture, team environment and management can have an effect on intervention uptake and thus influence the effectiveness. Previous studies revealed no differences for work environment and occupation, ${ }^{22}$ however we plan to control for this factor as a covariate. Furthermore, interventions will take place during working hours at the worksite. The participation of the nurses could be hindered by setting specific framework conditions. In order to keep the impairment as low as possible, prior organisation and consultation with the management will be crucial.

\section{Trial status}

The randomised controlled trial commenced in January 2018. The first subject was recruited on 13 August 2018. The anticipated date of recruitment completion is expected in December 2020.

\section{Author affiliations \\ ${ }^{1}$ Department of Human Movement Science, University of Hamburg, Hamburg, Germany \\ ${ }^{2}$ Department of Sport and Health, University of Paderborn, Paderborn, Germany ${ }^{3}$ Institute of Sports and Sports Science, Karlsruhe Institute of Technology, Karlsruhe, Germany \\ ${ }^{4}$ Institute of Medical Physics, Friedrich-Alexander University Erlangen-Nürnberg, Erlangen, Germany \\ ${ }^{5}$ Department of Sports and Exercise Science, University of Stuttgart, Stuttgart, Germany \\ ${ }^{6}$ Institute of Sport and Exercise Sciences, University of Münster, Münster, Germany ${ }^{7}$ Institute of Sports Sciences, Goethe University of Frankfurt, Frankfurt, Germany ${ }^{8}$ Institute of Human Movement Science and Health, Chemnitz University of Technology, Chemnitz, Germany \\ ${ }^{9}$ Biological Psychology and Neuroergonomics, Technical University of Berlin, Berlin, Germany}

Contributors This study was carried out in collaboration between all authors. BW had the project idea and is the head of the multicentre study. Study contents were additionally refined by BW, CH, CV-R, DS, LV, MW, NS and TJ, who are grant holders and principal investigators. All authors were involved in the design of the study protocol. BW and A-KO developed the intervention with revision of all authors A-KO wrote the first draft of the manuscript. JP wrote the intervention section. NS calculated the sample size and wrote the statistical section. A-KO, JP, L-MA, MB, LLB, CH, BJ, TJ, WK, TK, HK, JR, NS, DS, CV-R, OV, LV, MW, JW, KZ and BW have been involved in the drafting and contributed significantly to the revision of this manuscript and have given approval of the final manuscript.
Funding This study was funded by the health insurance Techniker Krankenkasse to the scientific concept. The study is part of the project 'Prevention and occupational health in long-term care' (PROCARE). Trial data will be analysed independently of the trial sponsors.

Disclaimer This funder will not play any role in the design of the study, data analysis, reporting of results, or the decision to present the manuscript for publication. The study protocol has not been reviewed by the funding body.

Competing interests None declared.

Patient consent for publication Not required.

Provenance and peer review Not commissioned; externally peer reviewed.

Open access This is an open access article distributed in accordance with the Creative Commons Attribution Non Commercial (CC BY-NC 4.0) license, which permits others to distribute, remix, adapt, build upon this work non-commercially, and license their derivative works on different terms, provided the original work is properly cited, appropriate credit is given, any changes made indicated, and the use is non-commercial. See: http://creativecommons.org/licenses/by-nc/4.0/.

ORCID iD

Ann-Kathrin 0tto http://orcid.org/0000-0003-4457-1625

\section{REFERENCES}

1 Ferrucci L, Guralnik JM, Studenski S, et al. Designing randomized, controlled trials aimed at preventing or delaying functional decline and disability in frail, older persons: a consensus report. J Am Geriatr Soc 2004;52:625-34.

2 Grobe T. Gesundheitsreport 2019: Pflegefall Pflegebranche? so geht's Deutschlands Pflegekräften. 2019, 2019.

3 Hasselhorn H-M, Tackenberg P, Müller BH. Vorzeitiger Berufsausstieg aus der Pflege in Deutschland als zunehmendes Problem für den Gesundheitsdienst - eine Übersichtsarbeit. Gesundheitswesen 2003;65:40-6.

4 Heinen MM, van Achterberg T, Schwendimann R, et al. Nurses' intention to leave their profession: a cross sectional observational study in 10 European countries. Int J Nurs Stud 2013;50:174-84.

5 Eriksen W, Bruusgaard D, Knardahl S. Work factors as predictors of intense or disabling low back pain; a prospective study of nurses aides. Occup Environ Med 2004;61:398-404.

6 Fiabane E, Giorgi I, Sguazzin C, et al. Work engagement and occupational stress in nurses and other healthcare workers: the role of organisational and personal factors. J Clin Nurs 2013;22:2614-24.

7 Magnavita N. Workplace violence and occupational stress in healthcare workers: a chicken-and-egg situation-results of a 6-year follow-up study. J Nurs Scholarsh 2014;46:366-76.

8 Meyer M, Wenzel J, Schenkel A. Krankheitsbedingte Fehlzeiten in der deutschen Wirtschaft im Jahr 2017. In: Badura B, Ducki A, Schröder $\mathrm{H}$, et al, eds. Fehlzeiten-Report 2018. Berlin, Heidelberg: Springer Berlin Heidelberg, 2018: 331-536.

9 Dawson AP, McLennan SN, Schiller SD, et al. Interventions to prevent back pain and back injury in nurses: a systematic review. Occup Environ Med 2007;64:642-50.

10 Simon M, Tackenberg P, Nienhaus A, et al. Back or neck-pain-related disability of nursing staff in hospitals, nursing homes and home care in seven countries--results from the European NEXT-Study. Int $J$ Nurs Stud 2008;45:24-34.

11 Semrau J, Hentschke C, Buchmann J, et al. Long-Term effects of interprofessional biopsychosocial rehabilitation for adults with chronic non-specific low back pain: a multicentre, quasiexperimental study. PLoS One 2015;10:e0118609.

12 Seidler A, Euler U, Bolm-Audorff U, et al. Physical workload and accelerated occurrence of lumbar spine diseases: risk and rate advancement periods in a German multicenter case-control study. Scand J Work Environ Health 2011;37:30-6.

13 Jensen LD, Gonge H, Jørs E, et al. Prevention of low back pain in female eldercare workers: randomized controlled work site trial. Spine 2006;31:1761-9.

14 Lorusso A, Bruno S, L'Abbate N. A review of low back pain and musculoskeletal disorders among Italian nursing personnel. Ind Health 2007;45:637-44.

15 Skargren E, Öberg B. Effects of an exercise program on musculoskeletal symptoms and physical capacity among nursing staff. Scand J Med Sci Sports 1996;6:122-30.

16 Horneij E, Hemborg B, Jensen I, et al. No significant differences between intervention programmes on neck, shoulder and low back 
pain: a prospective randomized study among home-care personnel. J Rehabil Med 2001;33:170-6.

17 Ewert T, Limm H, Wessels T, et al. The comparative effectiveness of a multimodal program versus exercise alone for the secondary prevention of chronic low back pain and disability. $\mathrm{Pm} R$ 2009;1:798-808.

18 Van Hoof W, O'Sullivan K, O'Keeffe M, et al. The efficacy of interventions for low back pain in nurses: a systematic review. Int $J$ Nurs Stud 2018;77:222-31.

19 Kolu P, Tokola K, Kankaanpää M, et al. Evaluation of the effects of physical activity, cardiorespiratory condition, and neuromuscular fitness on direct healthcare costs and Sickness-Related absence among nursing personnel with recurrent nonspecific low back pain Spine 2017;42:854-62.

20 Alexandre NM, de Moraes MA, Corrêa Filho HR, et al. Evaluation of a program to reduce back pain in nursing personnel. Rev Saude Publica 2001;35:356-61.

21 Otto A-K, Bischoff LL, Wollesen B. Work-Related burdens and requirements for health promotion programs for nursing staff in different care settings: a cross-sectional study. Int J Environ Res Public Health 2019;16:ijerph16193586.

22 Wollesen B, Menzel J, Lex H, et al. The BASE-Program-A multidimensional approach for health promotion in companies. Healthcare 2016;4:healthcare4040091.

23 Kreis J, Bödeker W. Gesundheitlicher und ökonomischer Nutzen betrieblicher Gesundheitsförderung und Prävention: Zusammenstellung Der wissenschaftlichen Evidenz. 1st ed, 2003.

24 Wollesen B, Lex H, Mattes K. BASE als Programm zur Prävention von (Rücken-)Beschwerden in der betrieblichen Gesundheitsförderung. Gruppendyn Organisationsberat 2012;43:389-411.

25 Schwarzer R. Modeling health behavior change: how to predict and modify the adoption and maintenance of health behaviors. Appl Psychol 2008;57:1-29.

26 Taylor N, Conner M, Lawton R. The impact of theory on the effectiveness of worksite physical activity interventions: a metaanalysis and meta-regression. Health Psychol Rev 2012;6:33-73.

27 Proper KI, Koning M, van der Beek AJ, et al. The effectiveness of worksite physical activity programs on physical activity, physical fitness, and health. Clin J Sport Med 2003;13:106-17.

28 Rongen A, Robroek SJW, van Lenthe FJ, et al. Workplace health promotion: a meta-analysis of effectiveness. Am J Prev Med 2013;44:406-15.

29 Healy G. Reducing prolonged sitting in the workplace: An evidence review. [Carlton South. VicHealth, 2012.

30 Chan A-W, Tetzlaff JM, Gøtzsche PC, et al. Spirit 2013 explanation and elaboration: guidance for protocols of clinical trials. BMJ 2013;346:e7586.

31 Cordes T, Bischoff LL, Schoene D, et al. A multicomponent exercise intervention to improve physical functioning, cognition and psychosocial well-being in elderly nursing home residents: a study protocol of a randomized controlled trial in the PROCARE (prevention and occupational health in long-term care) project. BMC Geriatr 2019;19:369.

32 Slesina W. FEBA: Fragebogen Zur subjektiven Einschätzung Der Belastung am Arbeitsplatz, 2009.

33 Caffier G, Steinberg U, Liebers F. Praxisorientiertes Methodeninventar Zur Belastungs- und Beanspruchungsbeurteilung Im Zusammenhang
MIT arbeitsbedingten Muskel-Skelett-Erkrankungen. New York: Verlag für neue Wissenschaft, 1999.

34 Crawford JO. The Nordic musculoskeletal questionnaire. Occup Med 2007:57:300-1

35 Bullinger M, Kirchberger I, Ware J. Der Deutsche SF-36 health survey Übersetzung und psychometrische Testung eines krankheitsübergreifenden instruments Zur Erfassung Der gesundheitsbezogenen Lebensqualität. $J$ Public Health 1995;3:21-36.

36 Ware JE, Kosinski M, Keller SD. SF-12: how to score the SF-12 physical and mental health summary scales. 4th ed. Lincoln, R.I, Boston, Mass: QualityMetric Inc; Health Assessment Lab, 2002.

37 Stieglitz R-D, Bullinger M, Kirchberger I. (1998). SF-36. Fragebogen zum Gesundheitszustand. Göttingen: Hogrefe. Preis DM 298. Zeitschrift für Klinische Psychologie und Psychotherapie. 1999;28:143-5.

38 Petrowski K, Paul S, Albani C, et al. Factor structure and psychometric properties of the trier inventory for chronic stress (tics) in a representative German sample. BMC Med Res Methodol 2012;12:42.

39 Schulz P, Schlotz W. Trierer Inventar Zur Erfassung von chronischem Streß (tics): Skalenkonstruktion, teststatistische Überprüfung und Validierung Der Skala Arbeitsüberlastung. Diagnostica 1999;45:8-19.

40 Voltmer E, Schwappach DLB, Frank E, et al. Work-Related behavior and experience patterns and predictors of mental health in German physicians in medical practice. Fam Med 2010;42:433-9.

41 Schaarschmidt U, Fischer AW. Arbeitsbezogenes Verhaltens- und Erlebensmuster. 3. überarbeitete und erweiterte Auflage. London: Pearson, 2008.

42 Weeks BK, Beck BR. The BPAQ: a bone-specific physical activity assessment instrument. Osteoporos Int 2008;19:1567-77.

43 WHO. Guideline. Geneva: World Health Organization, 2015.

44 Kessler RC, Barber C, Beck A, et al. The world Health organization health and work performance questionnaire (HPQ). J Occup Environ Med 2003;45:156-74.

45 Wollesen B, Menzel J, Drögemüller R, et al. Präventionsbedarf in der BGF von KMU - Querschnittsdaten des EU-Projekts „Fit for Business" (Teil 1). B \& G 2016;32:80-4.

46 Chen MJ, Fan X, Moe ST. Criterion-related validity of the Borg ratings of perceived exertion scale in healthy individuals: a meta-analysis. $J$ Sports Sci 2002;20:873-99.

47 Campbell MK, Elbourne DR, Altman DG, et al. Consort statement: extension to cluster randomised trials. BMJ 2004;328:702-8.

48 Faul F, Erdfelder E, Buchner A, et al. Statistical power analyses using G*Power 3.1: tests for correlation and regression analyses. Behav Res Methods 2009;41:1149-60.

49 Andersen HH, Mühlbacher A, Nübling M, et al. Computation of Standrad values for physical and mental health scale scores using the SOEP version of SF-12v2. Schmollers Jahrbuch, 2007: 171-82.

50 Wollesen B, Menzel J, Drögemüller R, et al. The effects of a workplace health promotion program in small and middle-sized companies: a pre-post analysis. J Public Health 2017;25:37-47.

51 Westermann C, Kozak A, Harling M, et al. Burnout intervention studies for inpatient elderly care nursing staff: systematic literature review. Int J Nurs Stud 2014;51:63-71. 